\title{
Indicadores emocionais no Desenho da Figura Humana: investigando evidências de validade
}

\author{
Ingrid PiccolloComparini' \\ Pontifícia Universidade Católica de Campinas, SP, Brasil \\ Solange Muglia Wechsler \\ Pontifícia Universidade Católica de Campinas, SP, Brasil \\ Wagner de Lara Machado \\ Pontifícia Universidade Católica de Campinas, SP, Brasil
}

\begin{abstract}
Resumo: O Desenho da Figura Humana (DFH) é muito utilizado para a avaliação emocional de crianças, porém há pouca validade empírica da medida. $O$ objetivo deste estudo foi investigar evidências de validade por critério externo para o DFH. A amostra foi composta de 91 crianças (62,6\% meninas), com idades entre II e I2 anos, que frequentavam instituições de educação básica. Para tanto, utilizou-se o DFH avaliado pelo sistema de dificuldades emocionais e Youth Self-Report (YSR), Inventário de Autoavaliação para Jovens que avalia dificuldades comportamentais e emocionais. Foram estimadas as correlações entre ambas as medidas e comparados os totais e os itens específicos do DFH com as dimensões comportamentais do YSR. Não foram observadas associações entre os totais de indicadores emocionais do DFH e nos totais do YSR. No presente estudo, não foi observada a validade do DFH como medida de problemas emocionais, indicando a necessidade de mais estudos sobre sua validade.
\end{abstract}

Palavras-chave: avaliação psicológica; desenho infantil; dificuldades emocionais; validade; problemas emocionais.

\section{EMOTIONAL INDICATORS IN HUMAN FIGURE DRAWING: INVESTIGATING} EVIDENCE OF VALIDITY

\begin{abstract}
The Human Figure Drawing (HFD) is widely used for emotional assessment of children, but there is little empirical validity of this measure. This study aims at investigating the validity evidence for external criteria of HFD. The sample consisted of 9I children (62.6\% girls), aged between II and I2 years, enrolled in elementary institutions. We used the HFD to evaluate emotional difficulties and Youth Self Report (YSR) system in assessing self-perception of psychological difficulties. The Pearson correlations between the two measures were estimated. We have compared the total and specific HFD items with the behavioral dimensions of YSR. We have observed no associations between complete emotional indicators of HFD and the total of YSR. In conclusion, the HFD was not validated as a measure of emotional issues, indicating the need for more studies.
\end{abstract}

Keywords: psychological assessment; children drawing; emotional difficulties; validity; emotional problems.

${ }^{1}$ Endereço de correspondência: Ingrid Piccollo Comparini: Rua Ana Jacinta de Andrade Couto, n. 51, Parque Industrial, Campinas, São Paulo. CEP: 13031-400; Tel.: (+5519) 988390195. E-mail: ingrid_comparini@hotmail.com; ingrid.comparini@gmail.com 
INDICADORES EMOCIONALES EN EL DISEÑO DE LA FIGURA HUMANA: INVESTIGACIÓN DE EVIDENCIAS DE VALIDEZ

\begin{abstract}
Resumen: La Figura dibujo Humana (FDH) es ampliamente utilizado para la evaluación emocional de los niños, pero hay poca validez empírica de la medida. El objetivo de este estudio fue investigar la evidencia de validez de los criterios externos para FDH. La muestra consistió en 9 I niños (62,6\% mujeres), con edades comprendidas entre los I I y los 12 años, asistiendo a las instituciones de educación básica. Para ello, se utilizó el FDH evaluados por las dificultades emocionales y sistema de Youth Self Report (YRS). Se estimaron las correlaciones entre las dos medidas y comparadas las partidas totales y específicas DFH con las dimensiones del comportamiento de YSR. No se observaron asociaciones entre los indicadores emocionales totales de FDH y el total de YSR. El presente estudio no mostró la validez del FDH como una medida de problemas emocionales, lo que indica la necesidad de más estudios sobre su validez.
\end{abstract}

Palabras clave: evaluación psicológica; los niños de dibujo; dificultades emocionales; validez; problemas emocionales.

\title{
Introdução
}

O uso do desenho enquanto forma de expressão humana remete aos primórdios da história (Menezes, Moré, \& Cruz, 2008). O interesse da Psicologia em aprofundar os trabalhos realizados com o desenho infantil data do final século XIX (Imuta, Scarf, Pharo, \& Hayne, 2013). O desenho passou a ser considerado não apenas forma de expressão humana, mas também foi ganhando notabilidade como forma de avaliação psicológica nos Estados Unidos (Handler \& Thomas, 2014) e em contextos internacionais (Dutta \& Sanyal, 2016). Suehiro, Benfica e Cardim (2016) apontaram em uma revisão de produção científica que estudos que têm focado a avaliação psicológica citam o Desenho da Figura Humana (DFH) como um dos instrumentos mais utilizados na área (independentemente de ordem classificatória) no contexto clínico, escolar e educacional. Além disso, o DFH é utilizado para avaliar aspectos de caráter emocional, cognitivo e criativo.

O uso do desenho da figura humana enquanto forma de avaliação emocional teve seu início em meados da década de 1930 e 1940. Os primeiros trabalhos que utilizavam o DFH tinham como matriz teórica construtos psicanalíticos (Segabinazi, 2010). Koppitz (1968) destaca que foi em 1949, com os estudos de Machover, que a análise por meio do desenho ganhou notabilidade. Machover defendia que era possível avaliar a personalidade da criança através da expressão gráfica. Apesar de grande precursora nos estudos do DFH enquanto modo de avaliação emocional, Machover recebeu críticas advindas da ausência de um sistema de tabulação padronizado e a validade do instrumento foi questionada.

No âmbito nacional, estudos dos indicadores emocionais ganharam forças a partir dos trabalhos realizados por Van Kolck em 1963. Ballas, Alves e Duarte (2011) apontaram que, para Van Kolck, o desenho estimulava o processo de organização de características internas de personalidade. Outro estudo de destaque foi elaborado por Koppitz (1968). A autora, partindo dos trabalhos de Goodenough e Machover, cons- 
truiu uma lista de indicadores emocionais para padronizar o que denominavam de avaliação emocional. Para a autora, itens que apresentassem frequência inferior a $16 \%$ na amostra estudada deveriam ser considerados indicadores emocionais presentes. A autora ainda apontou que a medida não deveria estar interligada com a maturação da criança.

Domingues, Alves, Rosa e Sargiani (2012) realizaram uma consideração fundamental acerca da compreensão do DFH. Apesar de o instrumento fornecer uma vasta gama de informações para que seja realizada a avaliação cognitiva, emocional e criativa, devem-se utilizar os indicadores estudados em pesquisas nacionais para realizar as análises que englobam o desenho. Por exemplo, Wechsler, Prado, Oliveira e Mazzarino (2011) estudaram 2.206 desenhos de crianças entre 5 e 11 anos utilizando o sistema proposto por Naglieri, McNeish e Bardos (1991) para a avaliação emocional. Nesse molde de avaliação, a criança deve elaborar três desenhos, uma figura masculina, uma feminina e uma de si própria. A avaliação se dava por uma soma total de pontos resultantes das três figuras elaboradas pela criança. As autoras ainda utilizaram o sistema de Wechsler, validado no âmbito nacional em 2003, para a avaliação cognitiva. As autoras observaram que o total de indicadores emocionais é influenciado significativamente por gênero e idade das crianças, tipo de figura desenhada e tipo de escola frequentada. As correlações entre aspectos cognitivos e emocionais foram negativas. Destacaram ainda a necessidade de cuidado na interpretação dos indicadores emocionais, uma vez que alguns indicadores poderiam refletir características do desenvolvimento cognitivo.

Embora tenha relevância e seja um dos instrumentos mais difundidos na prática de avaliação infantil, alguns autores questionam a validade do instrumento. Segabinazi e Bandeira (2012) apontam que não existem elementos que comprovem a validade empírica do DFH enquanto medida emocional. Devido a isso, autores como Lilienfeld et al. (2015) descrevem que o DFH abriga um lugar controverso no campo geral de avaliação.

Entretanto, apesar dessas considerações, vale destacar que a investigação sobre o Desenho da Figura Humana favorece o instrumento e promove discussões acerca de seu uso enquanto forma de avaliação psicológica. Suehiro (2013) fez uma consideração importante ao avaliar artigos publicados sobre o desenho entre 2002 e 2012. A autora destacou que, dentre os 32 artigos por ela analisados, somente quatro buscavam evidências de validade e precisão para o instrumento, constatando uma lacuna na área.

O DFH-emocional apresentou evidências positivas com resultados preliminares apresentados por Comparini e Wechsler em 2015. As autoras trabalharam com os indicadores emocionais propostos por Wechsler (2013) e avaliaram 166 crianças entre 5 e 13 anos ( $M=8$ anos), sendo 87 do gênero feminino e 79 do masculino. Destas, 64 crianças estavam institucionalizadas, 50 frequentavam clínicas-escola e 52 eram de escolas públicas. A partir das análises, as autoras descreveram diferenças de gênero e 
de grupos contrastantes. A análise post-hoc, para averiguar as distinções entre grupos, apontou que existiam diferenças significativas entre instituição e escola pública, mas não entre instituição e clínica escola. Tais dados indicariam evidências de validade no sistema dos indicadores emocionais. Além dos estudos com grupos contrastantes, outra forma de buscar evidências de validade para o DFH é por meio de comparações realizadas com outro instrumento já validado que também avalie dificuldades emocionais.

O estudo realizado por Dykens (1996) relacionou indicadores emocionais segundo o sistema de Naglieri com os do Children Behavior Checklist - CBCL (inventário de dificuldades emocionais que proporciona uma visão de múltiplos informantes). Ao contrário do esperado, os itens apresentaram baixas correlações.

Arteche (2006) também utilizou o CBCL e o correlacionou com o sistema de Koppitz para a análise de desenho. A autora trabalhou com crianças entre 9 e 12 anos. Os resultados apontaram correlações significativas entre o total de indicadores do DFH e fatores de Ansiedade/Depressão e Internalização avaliados no CBCL. Ainda foi constatado que itens como "Transparências" avaliados pelo DFH estariam relacionados a aspectos de avaliação emocional e discriminariam crianças com dificuldades emocionais.

Outro estudo comparativo entre o DFH seguindo os critérios de Koppitz e o CBCL é o trabalho de Segabinazi (2010). A autora contou com a avaliação de quatro juízes e examinou o DFH segundo as escalas globais de qualidade artística, normalidade e diferenciação sexual. Observaram-se resultados relevantes e significativos no que diz respeito aos índices de fidedignidade entre juízes acerca das escalas globais. Os resultados demonstraram direcionamentos positivos relacionados ao futuro do Desenho da Figura Humana nas escalas globais de avaliação emocional.

Assim, embora os resultados dos estudos descritos anteriormente no que diz respeito aos indicadores emocionais do desenho sejam contraditórios, é importante que estudos sejam produzidos nessa área para que a técnica consiga alcançar caráter de fidedignidade e validade. Finn (2012) faz um alerta importante sobre tais resultados contraditórios encontrados na literatura. Para o autor, existe uma ausência de relações entre instrumentos que pressupõe estilos de tarefas distintos. Por exemplo, a relação entre certos tipos de instrumentos, como testes de autorrelato, entrevistas ou testes de personalidade baseados em desempenho, possuem baixa relação entre si por serem tipos de avaliações e medições distintos; fato que pode ter influenciado os resultados encontrados descritos.

Entretanto, estudos como o Dykens (1996), de Arteche (2006) e de Segabinazi (2010) são relevantes ao seguir esse delineamento de pesquisa, no sentido de relacionar o Desenho da Figura Humana com questionários de comportamentos. Releva-se que não foram encontrados na revisão de literatura estudos que comparassem o DFH com questionários de autorrelato, que poderiam minimizar influências advindas dos relatos de terceiros. Nesse sentido, estudar as correlações do DFH avaliado pelo sistema emocional com um instrumento de autorrelato já validado no país, que também 
avalie dificuldades emocionais, é de suma importância e pode fornecer aspectos de validade do desenho da figura humana. Dessa forma, pensa-se que um diferencial para este estudo seja analisar as correlações entre DFH e Youth Self Report (YSR).

O YSR é um instrumento de autorrelato utilizado em larga escala que, assim como o $\mathrm{CBCL}$, faz parte do sistema Achenbach de avaliação. Foi validado para o âmbito nacional por Rocha (2012) em um estudo que contou com 540 crianças e adolescentes encaminhados ao serviço de clínica escola de psicologia e 2.836 que não foram encaminhados, ou seja, estudantes de escolas regulares brasileiras. Os jovens provinham de cinco regiões brasileiras e tinham entre 11 e 18 anos. A autora encontrou adequação do modelo fatorial do instrumento para a população brasileira (RMSEA $=0,032$ ), capacidade discriminante das escalas e dos itens de problemas emocionais/comportamentais e bons índices de consistência interna.

$\mathrm{Na}$ revisão literária, foram encontrados trabalhos que utilizaram o instrumento com objetivos de: identificar perfis psicológicos de adolescentes grávidas (Bernardo \& Oliveira-Monteiro, 2015); investigar a relação entre estresse e problemas de comportamento (Oliveira-Monteiro, 2012); avaliar a relação entre temperamento, enfrentamento e problemas emocionais (Justo \& Enumo, 2015); e ainda a correlação entre os instrumentos YSR e CBCL (Rocha, Ferrari \& Silvares, 2011). Apesar de os estudos encontrados serem importantes na área de avaliação psicológica, não foram localizados trabalhos que correlacionassem o YSR com o DFH.

Assim, o presente estudo teve como objetivo investigar evidências de validade por critério externo do DFH enquanto medida para avaliar dificuldades emocionais, correlacionando-o com o YSR, que é um instrumento de autorrelato já validado no país.

\section{Método}

\section{Participantes}

A amostra final deste estudo contou com 91 crianças, sendo 57 meninas $(62,6 \%)$ e 34 meninos (37,4\%), com idades entre $11(52,7 \%)$ e 12 anos (47,3\%). Destes, $43(47,3 \%)$ frequentavam a escola particular e $48(52,7 \%)$, a escola pública em uma cidade no interior de São Paulo.

\section{Instrumentos}

1) O Desenho da Figura Humana, segundo o sistema de indicadores emocionais proposto por Wechsler (2013), que se encontra em fase de validação, analisa aspectos do desenho como presença/ausência de membros e partes do corpo (cabeça, pernas, braços, nariz, boca, olhos etc.); seu formato (olhos fechados, dedos em formas de garras, figuras de perfil ou de costas); representações de agressividade (como presença de armas e facas) e a representação de nudez total ou parcial do desenho. Para isso, solicita-se à criança a elaboração de dois desenhos, um de uma figura masculina e um de 
uma figura feminina. Para a realização do desenho, a criança conta com duas folhas sulfite do tipo A4. Também é orientado para a criança que ela não pinte o desenho, ou seja, ela deve utilizar apenas lápis e borracha. O tempo médio de aplicação é de 30 minutos. Os desenhos são corrigidos de forma independente e contam com a avaliação de 59 itens para o desenho da figura masculina e 59 para a figura feminina. Esses itens estão agrupados em 16 dimensões. Conforme referendado anteriormente, o estudo elaborado por Comparini e Wechsler (2015) apresentou evidências de validade positivas para esse sistema de avaliação.

2) O YSR foi desenvolvido por Achenbach e Rescorla (2010) e pertence ao Sistema Achenbach de Avaliação Empiricamente Baseada (ASEBA - Achenbach System of Empirically Based Assessment). O sistema foi traduzido para 85 idiomas e engloba inventários que podem ser utilizados em diversas faixas etárias (Achenbach \& Rescorla, 2010).

O YSR engloba competências de caráter social e dificuldades comportamentais em participantes entre 11 e 18 anos. Pode ser dividido em duas partes, sendo a primeira voltada à análise de comportamentos sociais desejados, que compõe a escala de aspectos positivos, e a segunda destinada à avaliação de problemas emocionais e de comportamento. A lista que se refere a comportamentos sociais desejados conta com 14 itens, enquanto a lista referente à avaliação de problemas emocionais e comportamentais tem 105 itens. O objetivo do inventário é apresentar descrições padronizadas das competências e dos problemas emocionais e de comportamento contemplando as escalas de: 1) ansiedade/depressão (AnxDep); 2) retraimento/depressão (WithDrep); 3) queixas somáticas (SomComp)/4) problemas de sociabilidade (SocProb); 5) problemas de atenção (AttProb); 6) problemas com o pensamento (ThougProb); 7) comportamento de quebra de regras (RBreakBehav); 8) comportamento agressivo (AgBehav). Também são apresentados perfis de escalas orientados pelo DSM, tais como: 1) problemas afetivos (AffProb); 2) problemas de ansiedade (AnxProb); 3) problemas somáticos (SomProb); 4) problemas de deficit de atenção (AttDefHipProb); 5) problemas de oposição e desafio (OppDefProb); 6) problemas de conduta (CondProb); 7) problemas de obsessão e compulsão (PTSProb); 8) problemas de estresse pós-traumático (PTSProb). O instrumento propõe ainda fornecer um perfil denominado de Qualidades Positivas (PositQual). Segundo Rocha (2012), o tempo médio de aplicação para a população brasileira é de 30 minutos.

\section{Procedimentos}

Após a aprovação do Comitê de Ética em Pesquisa com seres Humanos, sob parecer de aprovação com CAAE 44590815.0.0000.5481, deu-se início as aplicações.

As aplicações ocorreram nas próprias escolas em pequenos grupos (entre 15 e 17 alunos). A aplicação dos dois instrumentos demandou dois encontros de 1 hora cada. Após essa etapa, as correções foram realizadas conforme o sistema proposto em cada 
instrumento. O DFH foi analisado segundo os critérios descritos por Wechsler (2013), e o YSR avaliado segundo os critérios propostos por Achenbach e Rescorla (2010), validados para o âmbito nacional por Rocha (2012). Com a finalidade de verificar a validade por critério externo da associação entre os instrumentos ou, ainda, as relações entre os resultados totais obtidos no DFH nos indicadores emocionais e no YSR, foi realizada a análise de correlação de Pearson.

Posteriormente, foi conduzida uma análise de rede para investigar o padrão de associação entre as variáveis investigadas. A análise de rede é uma técnica gráfica que combina algoritmos de associação (Machado, Vissoci \& Epskamp, 2015). Primeiro, foi estimada a matriz de correlações bivariadas e, em seguida, calculou-se a matriz de correlações parciais pelo método Graphical Leastand Absolut Shrinkageand Selection Operator (GLASSO), que penaliza o modelo anulando associações muito baixas, com o estimador de máxima verossimilhança e índice de ajuste Extended Bayesian Information Criteria. Essa matriz de associações é representada em um objeto gráfico que atende a alguns pressupostos. As variáveis são representadas como nodos e as relações entre elas são representadas por linhas verdes (positivas) e vermelhas (negativas). Após um estado inicial aleatório de repulsão, as variáveis são aproximadas conforme as associações entre elas (algoritmo de Fruchterman-Reingold). As variáveis que possuem o maior número de associações tendem a ficar no centro do grafo enquanto variáveis com poucas associações tendem a permanecer na margem. Para avaliar a rede, foram utilizadas além das associações entre as variáveis as medidas de conectividade (betweenness), proximidade (closeness) e força (strength), que representam, respectivamente, o quão cada variável conecta outros pares no sistema, o número de associações entre as demais variáveis e, por fim, a magnitude das associações que cada variável possui.

\section{Resultados}

A primeira análise se deu pelas correlações entre totais dos indicadores emocionais do DFH e totais obtidos no YSR. Uma vez que não foram encontradas relações significativas entre o total do DFH com os indicadores totais do YSR, a análise se seguiu a partir de um critério de eliminação de itens quando sua frequência fosse inferior a $10 \%$ no Desenho da Figura Humana. Isso ocorreu devido ao fato de ser observado que vários itens emocionais no DFH eram raros ou inexistentes. Dessa forma, foram retirados 35 itens da figura feminina e 34 da figura masculina. Assim, com o restante dos itens (total = 37 itens), foi composta uma medida denominada "itens reduzidos" e deu-se continuidade às análises de correlação, como apresentadas a seguir: 
Figura I. Matriz de correlações bivariadas entre as variáveis investigadas.

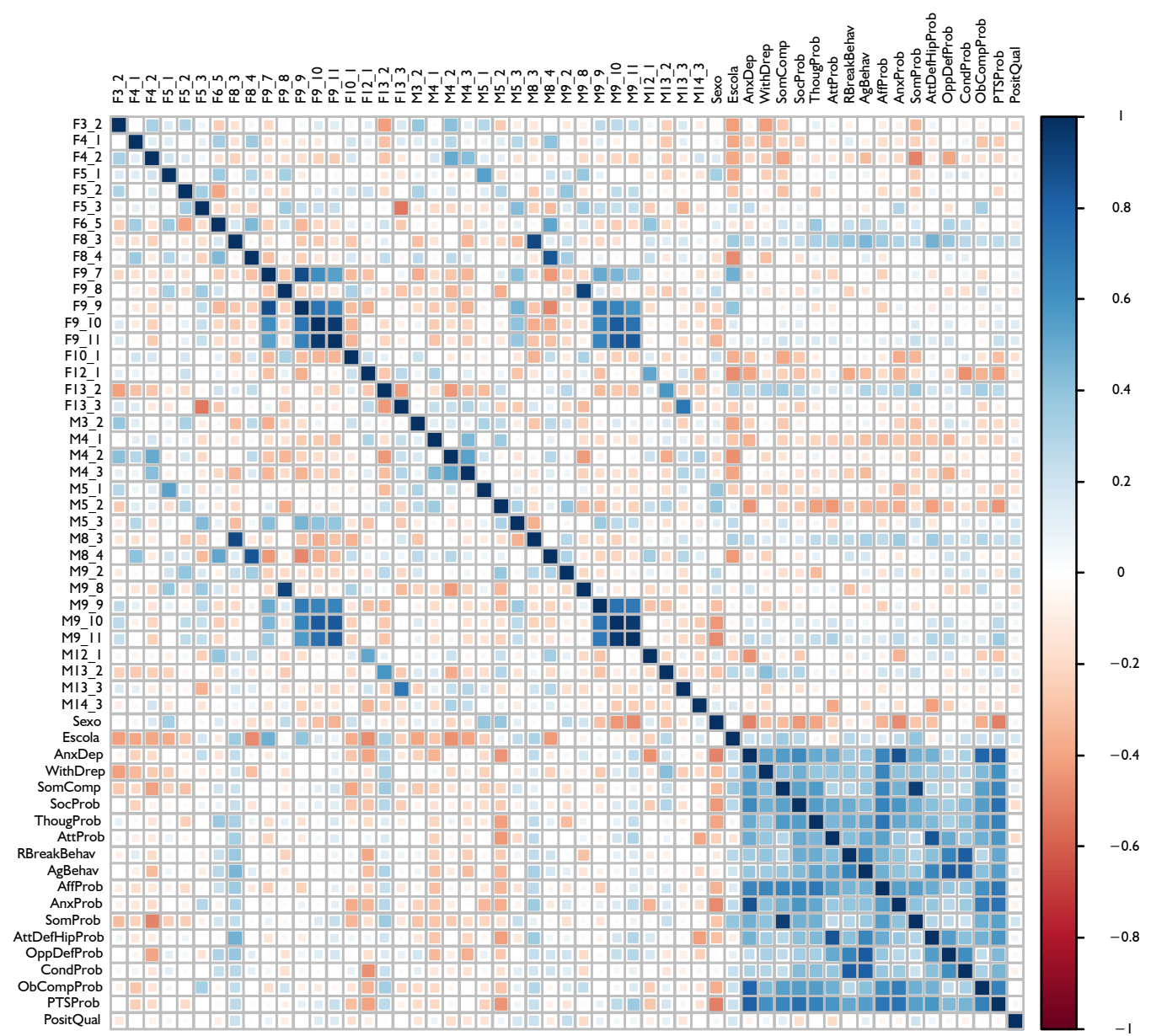

3.2 Figura Inclinada; 4.I Desintegração Cabeça-Pescoço; 4.2 Desintegração Braços-Tronco; 5.I Traço Reforçado; 5.2 Traço Borrado/ apagado; 5.3 Desistências; 6.5 Região Genital Sombreada; 8.3 Linha de base; 8.4 Letras e números; 9.7 Pernas Omitidas; 9.8 Nariz Omitido; 9.9 Pés Omitidos; 9.10 Mãos Omitidas; 9.II Dedos Omitidos; I0.I Cabeça Omitida; I2.I Transparência de roupas ou Objetos; 13.2 Formato Inclinado; I3.3 Olhar Desviado; I4.3 Presença de dentes;

*Itens com a letra 'F' são itens analisados através da figura feminina; Itens com a letra 'M' são itens da figura masculina. Fonte: Elaborado pelos autores.

A Figura 1 demonstra as correlações entre itens reduzidos do DFH e perfis individuais do YSR. As cores indicam a direção e intensidade das associações conforme legenda no lado direito da figura. Foi observada significância estatística quando $r \geq$ 0,20 , bicaudal. Vale destacar que não foram encontradas relações significativas entre o Total de Itens Reduzidos do DFH com os totais do YSR. Observam-se ainda 
poucas relações positivas significativas entre as outras dimensões dos dois instrumentos, ou seja, a maioria das relações positivas encontra-se entre os perfis analisados pelo YSR. Ainda assim, foram encontradas quatro dimensões que apresentassem relações positivas significativas. A dimensão "Linha de Base" avaliada no DFH apresentou correlações moderadas significativas positivas com Problemas de Pensamento, Comportamento de Quebra de Regras, Comportamento Agressivo, Problemas Externalizantes, Problemas de Deficit de Atenção e Hiperatividade, Problemas de Transtorno de Oposição e Desafio, Transtorno de Conduta e Total de Problemas do YSR. A dimensão "Genital Sombreada" avaliada pelo DFH apresentou correlações positivas porém fracas, com Problemas de Pensamento, Problemas Externalizantes e Transtorno de Oposição analisados pelo YSR. A dimensão "Desistência" do DFH apresentou correlações fracas com Problemas de Ansiedade, Problemas de Deficit de Atenção, Problemas de Obsessão e Compulsão e Ansiedade/Depressão avaliadas pelo YSR. Por fim, "Mãos Omitidas" do DFH apresentou correlações fracas positivas, com o perfil de estresse pós-traumático do YSR. Considerando as baixas correlações, decidiu-se realizar uma análise de rede para investigar o padrão de associação entre as variáveis investigadas.

Figura 2. Rede de correlações parciais entre as variáveis investigadas.

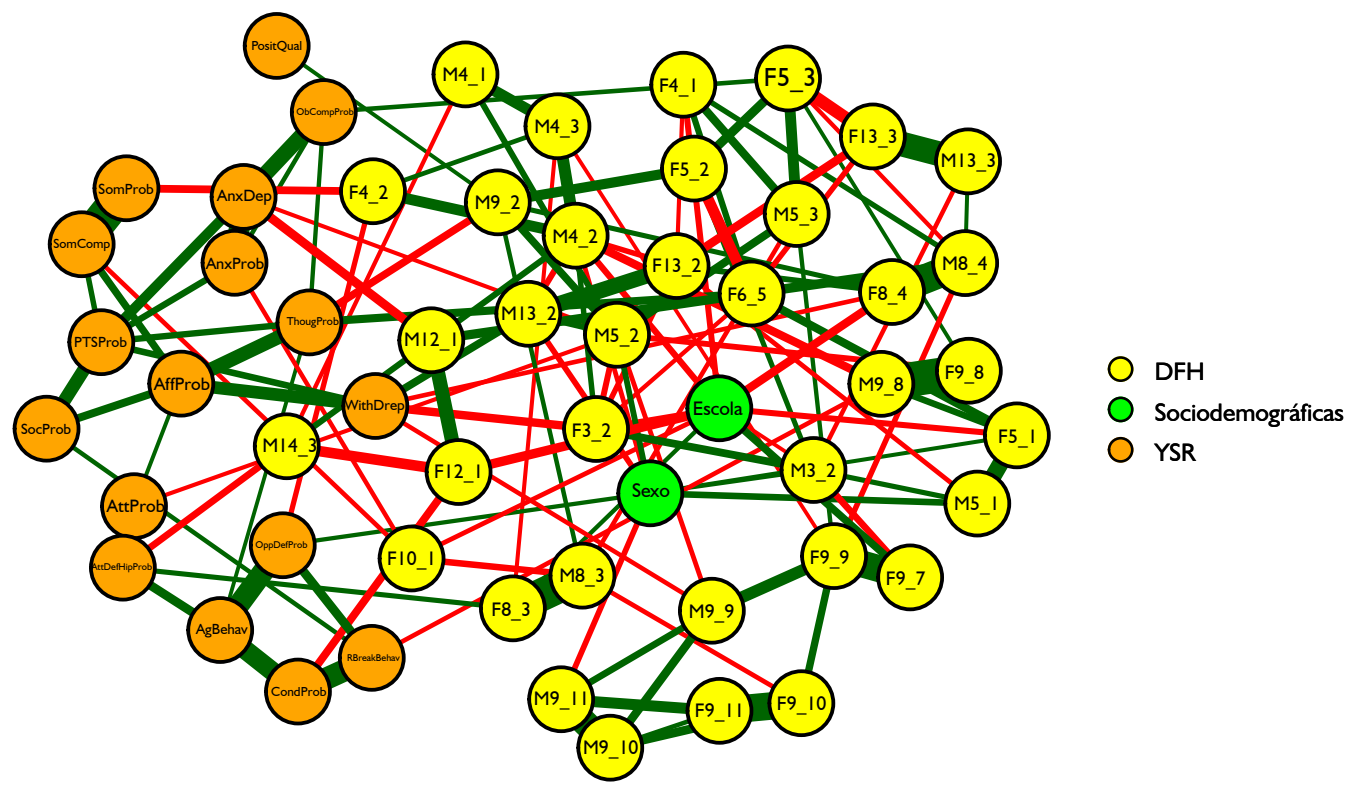

Fonte: Elaborada pelos autores. 
Cada nodo representa uma das variáveis: linhas verdes e vermelhas indicam a direção das associações e a espessura das linhas indicam a magnitude das mesmas associações.

A Figura 2 demonstra que dimensões analisadas pelo DFH mantêm pouca relação quando comparadas com o YSR. Os itens do DFH e YSR encontram-se, em sua maioria, em lados opostos, demonstrando a baixa correlação entre eles. O padrão de associação entre os dois instrumentos foi fraco e não apresentou nível de significância.

\section{Discussão}

Conforme descrito, o desenho enquanto forma de expressão humana é atualmente reconhecido como um dos principais métodos de avaliação psicológica (Menezes, Moré \& Cruz, 2008). Apesar de ser um dos instrumentos mais tradicionais na área de avaliação, o DFH ainda carece de comprovação empírica para a validação de seu sistema de indicadores emocionais.

O objetivo deste estudo foi buscar evidências de validade para o sistema de indicadores emocionais no Desenho da Figura Humana proposto por Wechsler (2013) através de critério externo. A revisão de literatura apontou estudos que comparassem o DFH com o $\mathrm{CBCL}$ - questionário respondido por responsáveis. Entretanto, não foram encontrados estudos que comparassem o desenho da figura humana com o YSR - respondido pelo próprio indivíduo.

Os resultados apontaram que as relações entre totais do DFH e do YSR variaram entre fracas e moderadas. Portanto, não foram verificadas evidências de validade por critério externo do sistema de indicadores emocionais. Considerando esse resultado, foi realizada uma análise de item por item. Essa análise de dimensões individuais do DFH com perfis analisados pelo YSR apresentou quatro correlações positivas significativas das dimensões presentes no DFH. São elas: "Linha de Base", "Genital Sombreada", "Desistência" e "Mãos Omitidas".

Arteche (2006) e Segabinazi (2010) sugeriram que apenas um determinado score de indicadores emocionais discriminaria as dificuldades da criança, ou seja, as autoras destacaram que apenas os resultados totais seriam relevantes para a avaliação de dificuldades emocionais através do DFH. No presente estudo, não foram encontradas relações entre os totais do DFH e os totais do YSR. Arteche (2006) ainda sugeriu que dimensões como "transparências" poderiam estar mais relacionadas com os indicadores emocionais do desenho, fato não encontrado no presente estudo.

Ao contrário do que era esperado, o perfil de Qualidades Positivas do YSR, que caracterizaria itens de indicadores de saúde mental e não de problemas emocionais, também não apresentou relações negativas significativas com as dimensões analisadas no sistema de dificuldades emocionais do Desenho da Figura Humana. Percebeu-se ainda na literatura uma lacuna relacionada à comparação da dimensão de "qualidades positivas" avaliada pelo YSR, o que evidenciou carência para a comparação com os dados encontrados neste estudo. 
Vale ainda destacar o baixo número de indicadores emocionais que apresentaram correlações positivas significativas. O sistema de Naglieri, McNeish e Bardos (1991) contava com 55 indicadores válidos na análise de dificuldades emocionais; o sistema de Koppitz (1968), por sua vez, apresentava 30 indicadores para a realização da análise, sendo ambos considerados sistemas clássicos de avaliação para dificuldades emocionais. Apesar de o sistema proposto por Wechsler (2013) apresentar 59 itens de avaliação, 37 contemplados na análise de Itens Reduzidos, neste estudo, apenas quatro apresentaram correlações significativas positivas.

Releva-se aqui que o delineamento metodológico utilizado neste trabalho não seguiu a mesma linha dos autores Segabinazi (2010) e Arteche (2006). Como citado anteriormente, ainda que esses estudos tenham colaborado com avanços importantes na área de avaliação psicológica, não foram encontrados na literatura estudos que correlacionassem o DFH-emocional com instrumentos de autorrelato. Nesse sentido, julga-se que o trabalho pode representar um diferencial na área. Entretanto, apesar de relevante, deve-se considerar que o DFH e o YSR possuem estilos de tarefas diferentes: o DFH é uma medida de expressão gráfica e o YSR é avaliado como medida de autorrelato. Esse fato, assim como apontado por Finn (2012), pode ter influenciado as análises e as correlações realizadas.

Itens individuais analisados do DFH, que podem estar associados às dificuldades emocionais e comportamentais, necessitam de maiores revisões e estudos com base empírica. Sugere-se que futuros estudos ampliem a amostra e analisem diferenças entre grupos para os indicadores emocionais do DFH. Por exemplo, os trabalhos apresentados por Comparini e Wechsler (2015) encontraram no sistema emocional proposto por Wechsler (2013) a sensibilidade do instrumento para discriminar grupos clínicos de estudantes em escolas regulares.

O presente trabalho também teve como limitação o fato de se atentar apenas às características emocionais expressas por meio do desenho. Outra sugestão para futuros estudos seria a utilização de um instrumento de controle para a avaliação de aspectos cognitivos e um instrumento para o controle de aspectos criativos que podem surgir no desenho e influenciar as análises finais. A influência de aspectos cognitivos no desenho analisado segundo o sistema de indicadores emocionais já havia sido alertada por Wechsler, Prado, Oliveira e Mazzarino (2011). Deve-se também expandir esse cuidado para as influências de aspectos criativos na interpretação dos indicadores emocionais.

\section{Conclusão}

Releva-se aqui o cuidado que o profissional deve manter ao realizar a avaliação a partir do DFH-emocional. O instrumento é importante e tem destaque na avaliação psicológica, em especial na avaliação psicológica infantil. Encontra-se no DFH a possibilidade de avaliar características emocionais, cognitivas ou criativas. Nesse sentido, a expressão 
gráfica do desenho da figura humana deve ser analisada com clareza e cautela pelo pesquisador ou psicólogo que realiza a avaliação. Deve-se compreender as potencialidades e as dificuldades da criança a partir de um contexto histórico vivenciado.

Recomendações finais alertam para a precaução na interpretação do desenho infantil como indicador de dificuldades emocionais. Apesar de essa estratégia ser bastante utilizada na avaliação infantil, estudos ainda são necessários para alcançar a validade dessa medida na avaliação emocional da criança. Deve-se ampliar as discussões sobre as possibilidades do DFH para a avaliação psicológica.

\section{Referências}

Achenbach, T. M., \& Rescorla, L. A. (2010). Mental Health Practitioners' Guide for the Achenbach System of Empirically Based Assessment (ASEBA) (7th ed.). Burlington, VT: University of Vermont, Research Center for Children, Youth \& Families.

Arteche, A. X. (2006) Indicadores emocionais no Desenho da Figura Humana: Construção e Validação de uma Escala Infantil. Programa de pós-graduação em psicologia. Universidade Federal do Rio Grande do Sul. Porto Alegre, Brasil.

Ballas, Y. G., Alves, I. C. B., \& Duarte, W. F. (2011). O desenho da figura humana de adolescentes portadores e não portadores de diabetes. Boletim de Psicologia, 61(134), 43-61.

Bernardo, J. L. \& Oliveira-Monteiro., N., R. (2015). Problemas emocionais e de comportamento em adolescentes grávidas. Psicologia Argumento, 33(81), 298-313.

Comparini, I. P., Wechsler, S. M., (2015). Indicadores emocionais no Desenho da Figura Humana em grupos contrastantes, 2015. Resumos do V Congresso de avaliação Psicológica.

Dykens, E. (1996). The draw-a-person task in persons with mental retardation: What does it measure? Research in Developmental Disabilities, 17(1), 1-13.

Domingues, S. F. S., Alves, I. C. B, Rosa, H. R., \& Sargiani R. A. (2012). As técnicas gráficas na avaliação cognitiva e da organização visomotora. In: Wechsler, S. M. Nakano, T. O Desenho infantil: Forma de expressão cognitiva, criativa e emocional. (pp. 97-126). São Paulo, SP: Casa do Psicólogo.

Dutta, M., \& Sanyal, N. (2016). A comparative study of emotional characteristics of children with and without ADHD by 'Draw a Man Test'. Journal of Projective Psychology \& Mental Health, 23(1), 27-33.

Finn, S. E. (2012). Implications of recent research in neurobiology for psychological assessment. Journal of Personality Assessment, 94(5), 440-449. 
Handler, L., \& Thomas, A.D. (Eds.). (2014). Drawings in assessment and psychotherapy: Research and application. New York: Routledge.

Imuta, K., Sacarf, D., Pharo, H., \& Hayne, H. (2013). Drawing a close to the use of human figure drawings as a projective measure of intelligence. Plus One, 8(3), 1-8.

Justo, A. P., \& Enumo, S. R. F. (2015). Problemas emocionais e de comportamento na adolescência: o papel do estresse. Boletim - Academia Paulista de Psicologia, 35(89), 350-370.

Koppitz, E. M. (1968). Psychological evaluation of children's human figure drawings. Grune \& Stratton: Nova York.

Lilienfeld, S. O., Lynn, S. J., \& Lohr, J. M. (2015). Science and pseudoscience in clinical psychology (2nd ed.). New York: Guilford Press.

Menezes, M., Moré, C. L. O. O., \& Cruz, R. M. (2008). O desenho como instrumento de medida de processos psicológicos em crianças hospitalizadas. Avaliação Psicológica, 7(2), 189-198.

Naglieri, J. A., McNeish, T. J., \& Bardos, A. N. (1991). Draw a person: screening procedure for emotional disturbance. Texas: Pro-ed.

Rocha, M. M., Ferrari, R. A., \& Silvares, E. F. M. (2011). Padrões de concordância entre múltiplos informantes na avaliação dos problemas comportamentais de adolescentes: implicações clínicas. Estudos e Pesquisas em Psicologia, 11(3), 948-964.

Rocha, M. M. (2012). Evidências de validade do "Inventário de Autoavaliação para adolescentes" (YSR/2001) para a população brasileira. Tese de doutorado. Programa de Pós-Graduação em Psicologia, Instituto de Psicologia da Universidade de São Paulo, SP, Brasil.

Segabinazi, J. D. (2010). Desenho da Figura Humana: Evidências de validade de escalas globais de avaliação. (Dissertação de mestrado). Recuperado de: http:// www.bibliotecadigital.ufrgs.br/da.php?nrb=000765803\&loc $=2011$

Segabinazi, J. D., \& Bandeira, D. R. (2012). Desenho da figura humana para avaliação emocional de crianças: evidências de validade de escalas globais. In Wechsler, S. M., \& Nakano, T. C. O desenho Infantil: forma de expressão cognitiva, criativa e emocional (pp. 149-175). São Paulo: Casa do Psicólogo.

Suehiro, J. D. (2013). Produção científica sobre o teste de Cloze. Psicologia Escolar e Educacional, 17(1), 223-232.

Suehiro, C. B., Benfica, T. S., \& Cardim, N. A. (2016). Produção Científica sobre o Teste Desenho da Figura Humana entre 2002 e 2012. Psicologia: Ciência e Profissão, 36(2), 439-448. 
Wechsler, S. M., Prado, C. M., Oliveira, K. S., \& Mazzarino, B. G. (2011). Desenho da Figura Humana: Análise da prevalência de indicadores emocionais. Psicologia, Reflexão e Crítica, 24(3), 411-418.

Wechsler, S. M. (2013). Triagem Emocional - DFH, Guia de correção. Manual Piloto. Campinas: LAMP/PUC-Campinas

Machado, W. L., Vissoci, J., \& Epskamp, S. (2015). Análise de rede aplicada à Psicometria e à Avaliação Psicológica. In C. S. Hutz, D. R. Bandeira, \& C. M. Trentini (Orgs.). Psicometria (pp. 125-146). Porto Alegre: ArtMed.

Submissão: 18.3 .2016

Aceite: 27.6.2017 